\title{
Microstructural Analysis of Cardiac Endomyocardial Biopsies with Synchrotron Radiation-Based X-Ray Phase Contrast Imaging
}

\author{
Hector Dejea ${ }^{1,2(\bowtie)}$, Patricia Garcia-Canadilla ${ }^{1,2}$, Marco Stampanoni ${ }^{1,3}$, \\ Monica Zamora ${ }^{4}$, Fatima Crispi ${ }^{4,5}$, Bart Bijnens ${ }^{2,6}$, and Anne Bonnin ${ }^{1}$ \\ 1 Swiss Light Source, Paul Scherrer Institut (PSI), 5232 Villigen, Switzerland \\ Hector.Dejea@psi.ch \\ 2 Physense, DTIC, Universitat Pompeu Fabra, 08018 Barcelona, Spain \\ ${ }^{3}$ Institute for Biomedical Engineering, ETH Zürich, 8092 Zurich, Switzerland \\ 4 Barcelona Center for Maternal-Fetal and Neonatal Medicine (BCNatal), \\ Hospital Clinic and Hospital Sant Joan de Deu, Barcelona, Spain \\ ${ }^{5}$ Centre for Biomedical Research on Rare Diseases (CIBER-ER), \\ Hospital Clinic, Barcelona, Spain \\ ${ }^{6}$ Institució Catalana de Recerca i Estudis Avançats (ICREA), Barcelona, Spain
}

\begin{abstract}
Nowadays, unexplained cardiovascular diseases (CVD) and heart transplant response are assessed by qualitative histological analysis of extracted endomyocardial biopsies (EMB), which is a time consuming procedure involving structural damage of the tissue and the analysis in only a few slices of a $3 \mathrm{D}$ structure. In this paper we propose synchrotron radiation-based X-ray phase contrast imaging (X-PCI) as a suitable technique for the analysis of different cardiac microstructures, such as collagen matrix, cardiomyocytes and microvasculature, and how they are affected in abnormal conditions. Following an established procedure in clinics, biopsies from Wistar Kyoto rats are extracted, imaged with X-PCI, and processed in order to show that the quantification of the endomysial collagen matrix, cardiomyocytes and microvasculature is possible, thus demonstrating that the intrinsic properties of X-PCI make it a powerful technique for cardiac microstructure imaging and a promising methodology for a faster and more accurate EMB analysis for CVD diagnosis and evaluation.
\end{abstract}

Keywords: Cardiac microstructure - Cardiac endomyocardial biopsy • $\mathrm{X}$-ray tomography $\cdot$ Phase contrast imaging

\section{Introduction}

The heart anatomy has been an important research target for centuries, which has led to a good understanding at macroscopic level. Nevertheless, the architecture at individual cell scale within the whole heart as well as its relationship and effects on the contractile mechanism remain still unclear. This lack of knowledge, together with the fact that cardiovascular diseases are the first cause of mortality

(C) Springer International Publishing AG 2017

M. Pop and G.A. Wright (Eds.): FIMH 2017, LNCS 10263, pp. 23-31, 2017.

DOI: $10.1007 / 978-3-319-59448-4 \_3$ 
in the world [1], are the reasons why a big effort from the scientific community is done towards obtaining a detailed multiscale description of the heart structure and function.

The cardiomyocytes are the contractile cells of the heart, with a size of approximately 10-20 $\mu \mathrm{m}$ diameter and 100-120 $\mu \mathrm{m}$ length, arranged in fibre-like structures (myofibres). These structures, in turn, are organized and oriented in a special manner in order to maximize the contractile function of the heart. In addition to the myocytes, a matrix of mainly type I fibrillar collagen $(\sim 85 \%)$ is present in the extracellular space of the myocardium, maintained by resorption and synthesis processes carried out by cells called fibroblasts. This collagen matrix is characterized by three different layers: endomysium (around cardiomyocytes), perimysium (separates myofibres) and epimysium (around groups of myofibres). The main function of the collagenous matrix is to serve as scaffold for myocyte alignment and to avoid an overstretching of the sarcomeres. Furthermore, it has secondary functions related to the functionality, electrical behaviour and vasomotor reactivity of the myocardium and its structures [2]. All these tissue components may change in shape, size or density under the effects of cardiac disorders, such as myocardial infarction, hypertension or transplant rejection $[3,4]$.

Endomyocardial biopsies (EMB) are often performed in clinics to evaluate rejection of heart transplants as well as to diagnose cardiovascular diseases that can not be assessed by means of diagnostic non-invasive imaging techniques, such as ultrasound or magnetic resonance [5-7]. EMB are usually qualitatively analyzed with histological procedures, which involve sample preparation processes that lead to tissue damage and alteration of the internal structure. In addition, only $2 \mathrm{D}$ information from a few slices is used for the analysis of the whole $3 \mathrm{D}$ sample.

In order to understand the microstructural organization within the heart with minimum tissue alteration, non-destructive imaging techniques with resolution at micrometre scale are needed. Current imaging techniques provide either high resolution (down to $155 \mathrm{~nm}$ pixel size and $1 \mu \mathrm{m}$ z-step) with a small field of view $\left(\sim 160 \times 160 \mu \mathrm{m}^{2}\right)$ and keep altering the sample [8,9], or a whole heart acquisition without enough resolution to resolve individual structures $[10,11]$.

Synchrotron radiation-based X-ray phase contrast imaging (X-PCI) is a recently emerged technique with potential to overcome the aforementioned limitations. In addition to the typical absorption imaging, X-PCI exploits the differences in refractive index between different materials, which improve the contrast while keeping the same X-ray dose. Among the different techniques exploiting the phase contrast effects, propagation-based imaging, used in this study, is of great interest for biomedical soft-tissue research [12]. In a recent study, phase contrast images of cardiac tissue have been obtained with a pixel size of $3.5 \mu \mathrm{m}$ with the use of this technique [13].

In this study, we propose a high-resolution procedure to assess the detailed cardiac microstructure in EMB biopsies from rat models, thus allowing the segmentation and quantification of cardiac tissue components. 


\section{Materials and Methods}

\subsection{Sample Preparation}

For this study, three cardiac biopsies of the basal septum, lateral wall and apex of the left ventricle (Fig. 1), with an approximate size of $2 \times 2 \times 4 \mathrm{~mm}^{3}$, were extracted from two 12 weeks old male control Wistar Kyoto rats (WKY) and fixed in $4 \%$ paraformaldehyde. The biopsies were then placed in thin-walled borosilicate glass tubes of $2 \mathrm{~mm}$ of inner diameter using $70 \%$ ethanol as medium, and mounted on the sample stage for image acquisition.

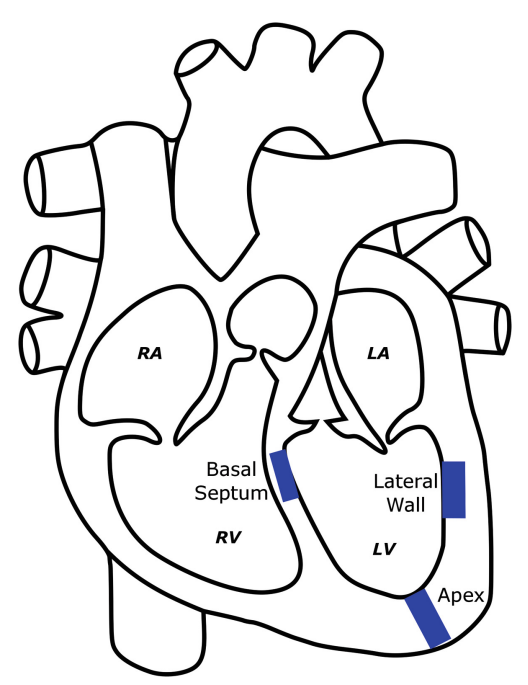

Fig. 1. Extracted biopsies for each of the hearts: basal septum, lateral wall and apex of the left ventricle.

\subsection{Data Acquisition}

The synchrotron-based X-ray tomography campaign was performed at the TOMCAT beamline (X02DA) of the Swiss Light Source (Paul Scherrer Institute, Switzerland). Propagation-based X-PCI was achieved with an X-ray beam of 20 $\mathrm{keV}$, a voxel size of $0.65 \mu \mathrm{m}$ and propagation distance of $20 \mathrm{~cm}$. Since the samples were larger than the field of view $\left(1.6 \times 1.44 \mathrm{~mm}^{2}\right)$, three volumes from each biopsy were imaged using a LuAG:Ce $20 \mu \mathrm{m}$ scintillator coupled to a PCO.Edge 5.5 CMOS Camera. For each volume, 2501 projections, 20 darks and 50 flats were acquired in approximately $7 \mathrm{~min}$ acquisition time. The so called darks are projections taken without beam exposure, used in order to correct for the detector's electronic noise, while flats are direct projections of the beam without sample, so as to correct for non-uniformities of the beam and imperfections in the optical components [14]. A sketch of the experimental setup can be found in Fig. 2. 


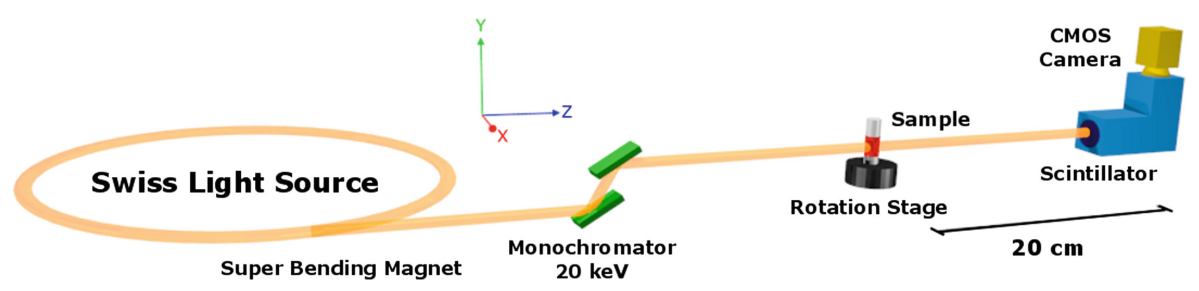

Fig. 2. Sketch of the experimental setup at the TOMCAT beamline at the Swiss Light Source

\subsection{Image Processing}

The acquired projections were reconstructed using the Gridrec algorithm [15]. Current detector technologies only allow intensity measurements, thus losing the information coded in the phase of the signal. Nevertheless, the phase information can be retrieved by several algorithms incorporating knowledge on wave propagation, such as the single distance phase retrieval method developed by Paganin [16]. Therefore, the data was reconstructed both with and without phase retrieval in order to later be able to fuse information from both phase and intensity images, respectively. The $\delta / \beta$ ratio used in the Paganin algorithm was 56.9. Then, representative $300 \times 300 \times 300 \mu^{3}$ subvolumes were cropped for processing.

For the segmentation task, the chosen tool was the open-source software Ilastik [18], based on interactive machine and active learning classifiers. The training process consisted on the iterative labelling of the images for cells, endomysial collagen and background in the three directions (2 slices per direction). Since the imaging conditions were the same for all samples, Ilastik was able to segment new input images with just small supervision (a few annotations in 1-2 slices) even if they did not correspond to the training datasets. The phase retrieved images were used to segment cells and background due to the increased contrast given by the Paganin approach. Nevertheless, the contrast of the image areas corresponding to the collagen matrix was reduced in most cases, so the non-phase retrieved images were used to segment it, as they are sharper and show a greater intensity difference compared to the rest of structures. Finally, objects smaller than 150 pixels in 3D were removed to reduce noise, both masks were fused and percentage of endomysial collagen per unit of cell area was computed. Due to acquisition problems, an apical biopsy dataset could not be used for analysis.

Moreover, we were also able to segment part of the cardiac vasculature with the Carving module included in Ilastik, based on seeded watershed algorithm where the seeds are given interactively by the user. In this case, the phase retrieved images were used, again due to the higher contrast between the vasculature wall and background. 


\section{Results}

The results obtained are represented for different rats, cardiac areas and volume sizes in order to show representative examples for each of the aforementioned acquisition and image processing procedures.

Two sets of orthogonal views of a representative subvolume are shown in Fig. 3, both with and without phase retrieval. Note how individual cardiomyocytes and its fibre-like arrangement can be distinguished. The brighter intensity spots correspond to the collagen matrix.
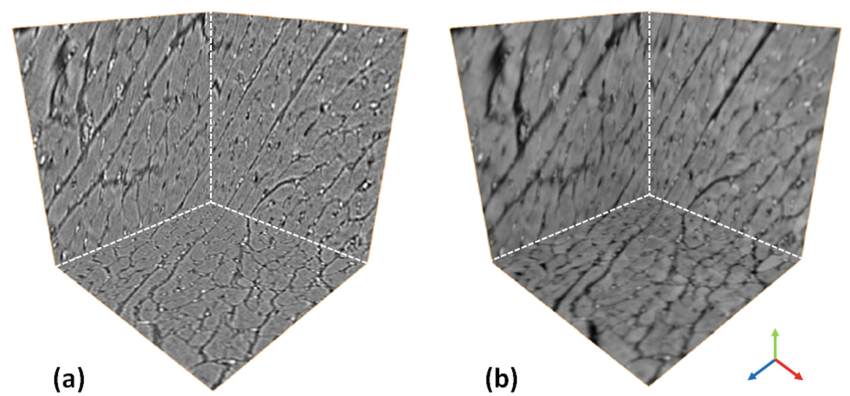

Fig. 3. Three orthogonal views (a) without and (b) with phase retrieval, corresponding to a $300 \times 300 \times 300 \mu^{3}$ subvolume of a biopsy of the left ventricle lateral wall of a WKY rat.

Figure 4 shows representative images of the segmentation procedure for the collagen matrix and cells. The 3D representation of the collagen matrix in Fig. $4 \mathrm{f}$ shows its organization in fibre-like structures following the direction of the cardiomyocytes. The relative amount of endomysial collagen within the different processed subvolumes for the WKY rats is detailed in Table 1. Finally, Fig. 5 shows the results of the vascular segmentation, where its branching morphology can be clearly observed.

\section{Discussion}

In this study we demonstrate that X-PCI, specifically in free-space propagation mode, can be used in order to assess the organization of the cardiac tissue at micrometer scale. In order to do so, images with a voxel size of $0.65 \mu \mathrm{m}$ have been acquired, thus improving the state-of-the-art $3.5 \mu \mathrm{m}$ for this imaging technique in the study of cardiac tissue [13]. Such improvement in resolution allows to analyse the samples by directly looking at its micrometer level structures, such as cardiomyocytes, endomysium network or microvasculature, which for the best of our knowledge, has never been achieved before with the use of X-ray imaging techniques. 

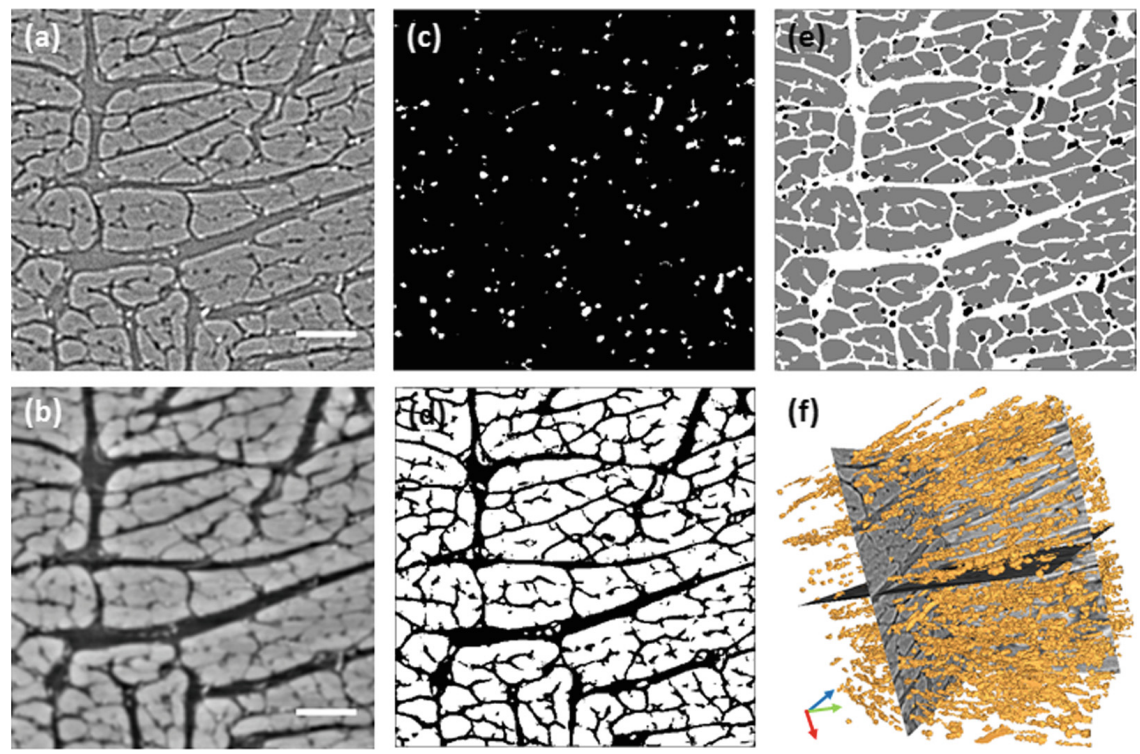

Fig. 4. Representation of the different steps in the segmentation of cardiomyocytes and collagen for a $300 \times 300 \times 300 \mu^{3}$ subvolume of the basal septum biopsy of a WKY rat. Scale bar corresponds to $50 \mu \mathrm{m}$ (a) Non-phase retrieved image. (b) phase retrieved image. (c) collagen segmentation. (d) cardiomyocytes segmentation. (e) resulting fusion of segmentations. (f) $3 \mathrm{D}$ rendering of the endomysial collagen segmentation in the entire subvolume.

Table 1. Calculated values for the endomysial collagen percentage in each of the biopsies.

\begin{tabular}{l|l|l|l}
\hline & Apex & LV Wall & Septum \\
\hline WKY1 & $4.23 \%$ & $6.37 \%$ & $4.60 \%$ \\
\hline WKY2 & - & $5.14 \%$ & $1.64 \%$ \\
\hline
\end{tabular}

In Table 1 it is shown that the collagen percentage is higher in the left ventricular wall than in the rest of areas. Moreover, the collagen percentage difference between rats in the left ventricular wall is in a smaller range $(\sim 1 \%)$ in comparison to the basal septum region $(\sim 2 \%)$. In the literature [17], endomysial collagen fraction with respect to total area was quantified from confocal microscopy images $(0.4 \mu \mathrm{m}$ voxel size). Left ventricular midwall WKY samples were analysed resulting in a $\sim 2.5 \%$ of collagen. In our case, similar but slightly higher values are observed in the left ventricular wall. The reasons for this increase are several. First, the calculation of collagen percentage is done with respect to cardiomyocyte area and not total area, which is more significant taking into account that endomysium is found around individual cells. In addition, the voxel size used 

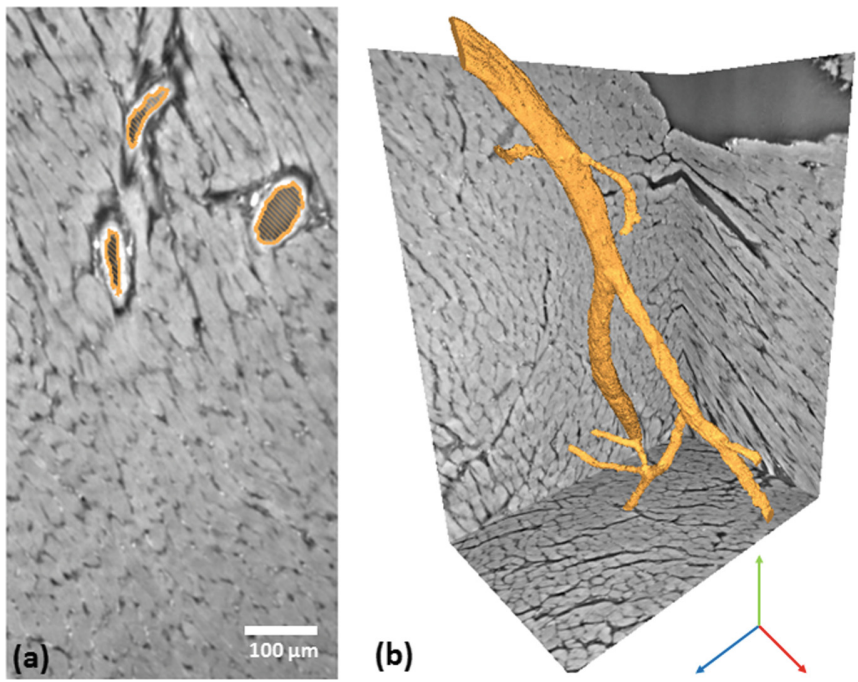

Fig. 5. (a) Selected slice and (b) 3D rendering of the vasculature segmentation in a $680 \times 920 \times 440 \mu^{3}$ subvolume of the basal septum biopsy of a WKY rat.

in X-PCI is larger than in the confocal microscopy technique used in the compared study, thus introducing a smoothing effect that may increase the amount of collagen quantified. Finally, as a limitation of the methodology, we would like to mention that platelets and collagen have very similar characteristics in the images and, therefore, when platelets are present in very small diameter vessels it is currently very difficult to differentiate them. Therefore, in order to overcome this limitation as much as possible, the analysis was performed in subvolumes without presence of visible vasculature.

The data obtained is of high interest in scientific and clinical terms, as it allows to characterize the tissue by direct observation of its main structural components to better understand how they influence the macroscopic organization and functionality. This means that we can also detect how these microstructures are affected under the influence of certain cardiac diseases or disorders, the so called cardiac remodelling, and thus comprehend in more detail the different abnormalities at all scales.

Nowadays, several diseases involving cardiac remodelling are assessed by histological imaging of extracted EMB. During such techniques, the biopsies undergo a series of destructive procedures that change the properties of the tissue and the final images are usually 2-dimensional, which can be overcome by the non-destructiveness of X-PCI and improved by the 3-dimensional time-efficient nature of the technique. In addition, the proposed image processing framework allows the quantitative evaluation of the acquired X-ray images, thus improving the accuracy of the currently qualitative assessment of histological images. 


\section{Conclusion}

The results show that X-PCI is a very promising technique for the analysis of the cardiac microstructure, as it is a 3-dimensional time-efficient non-destructive technique that allows the detailed observation of the different elements of the tissue at micrometer level. Therefore, the development and translation of X-PCI to a table-top system has the potential to improve the biopsy-based diagnostic procedures thanks to easier, less aggressive and shorter preparation and imaging procedures.

Acknowledgments. This research has received funding from the EU FP7 for research, technological development and demonstration under grant agreement VP2HF (no. 611823) and from the Spanish Ministry of Economy and Competitiveness (gTIN2014-52923-R, the Maria de Maeztu Units of Excellence Programme MDM2015-0502) and FEDER. P.G.C. wants to acknowledge EMBO for the short-term fellowship to do her research stay at X-ray Tomography group in Paul Scherrer Institut (PSI).

\section{References}

1. World Health Organization (WHO): Cardiovascular Diseases (CVDs). http:// www.who.int/mediacentre/factsheets/fs317/en/. Accessed 11 May 2016

2. Weber, K.T., Sun, Y., Bhattacharya, S.K., Ahokas, R.A., Gerling, I.C.: Myofibroblast-mediated mechanisms of pathological remodelling of the heart. Nat. Rev. Cardiology 10(1), 15-26 (2013)

3. Burchfield, J.S., Xie, M., Hill, J.A.: Pathological ventricular remodeling mechanisms: part 1 of 2. Circulation 128(4), 388-400 (2013)

4. Cohn, J.N., Ferrari, R., Sharpe, N.: Cardiac remodeling-concepts and clinical implications: a consensus paper from an international forum on cardiac remodeling. J. Am. Coll. Cardiol. 35(3), 569-582 (2000)

5. Dujardin, K.S., Enriquez-Sarano, M., Rossi, A., Bailey, K.R., Seward, J.B.: Echocardiographic assessment of left ventricular remodeling: are left ventricular diameters suitable tools. J. Am. Coll. Cardiol. 30(6), 1534-1541 (1997)

6. Vogel-Claussen, J., Rochitte, C.E., Wu, K.C., Kamel, I.R., Foo, T.K., Lima, J.A.C., Bluemke, D.A.: Delayed enhancement MR imaging: utility in myocardial assessment 1. Radiographics 26(3), 795-810 (2006)

7. From, A.M., Maleszewski, J.J., Rihal, C.S.: Current status of endomyocardial biopsy. Mayo Clin. Proc. 86(11), 1095-1102 (2011)

8. Tobita, K., Garrison, J.B., Liu, L.J., Tinney, J.P., Keller, B.B.: Three-dimensional myober architecture of the embryonic left ventricle during normal development and altered mechanical loads. Anat. Rec. Part A Discoveries Mol. Cell. Evol. Biol. 283(1), 193-201 (2005)

9. Jouk, P., Mourad, A., Milisic, V., Michalowicz, G., Raoult, A., Caillerie, D., Usson, Y.: Analysis of the fiber architecture of the heart by quantitative polarized light microscopy. Accuracy, limitations and contribution to the study of the fiber architecture of the ventricles during fetal and neonatal life. Eur. J. Cardiothorac. Surg. 31(5), 915-921 (2007) 
10. Schmid, P., Jaermann, T., Boesiger, P., Niederer, P.F., Lunkenheimer, P.P., Cryer, C.W., Anderson, R.H.: Ventricular myocardial architecture as visualised in postmortem swine hearts using magnetic resonance diffusion tensor imaging. Eur. J. Cardiothorac. Surg. 27(3), 468-472 (2005)

11. Lombaert, H., Peyrat, J., Croisille, P., Rapacchi, S., Fanton, L., Cheriet, F., Clarysse, P., Magnin, I., Delingette, H., Ayache, N.: Human atlas of the cardiac fiber architecture: study on a healthy population. IEEE Trans. Med. Imaging 31(7), 1436-1447 (2012)

12. Cloetens, P., Ludwig, W., Baruchel, J., Guigay, J., Pernot-Rejmánková, P., SaloméPateyron, M., Schlenker, M., Buffière, J., Maire, E., Peix, G.: Hard x-ray phase imaging using simple propagation of a coherent synchrotron radiation beam. J. Phys. D Appl. Phys. 32(10A), A145 (1999)

13. Mirea, I., Varray, F., Zhu, Y.M., Fanton, L., Langer, M., Jouk, P.S., Michalowicz, G., Usson, Y., Magnin, I.E.: Very high-resolution imaging of post-mortem human cardiac tissue using X-ray phase contrast tomography. In: van Assen, H., Bovendeerd, P., Delhaas, T. (eds.) FIMH 2015. LNCS, vol. 9126, pp. 172-179. Springer, Cham (2015). doi:10.1007/978-3-319-20309-6_20

14. Seibert, J.A., Boone, J.M., Lindfors, K.K.: Flat-field correction technique for digital detectors. Proc. SPIE Med. Imaging 1998(3336), 348-354 (1998)

15. Marone, F., Stampanoni, M.: Regridding reconstruction algorithm for real-time tomographic imaging. J. Synchrotron Radiat. 19(6), 1029-1037 (2012)

16. Paganin, D., Mayo, S.C., Gureyev, T.E., Miller, P.R., Wilkins, S.W.: Simultaneous phase and amplitude extraction from a single defocused image of a homogeneous object. J. Microsc. 206(1), 33-40 (2002)

17. LeGrice, I.J., Pope, A.J., Sands, G.B., Whalley, G., Doughty, R.N., Smaill, B.H.: Progression of myocardial remodeling and mechanical dysfunction in the spontaneously hypertensive rat. Am. J. Physiol. Heart Circulatory Physiol. 303(11), H1353-H1365 (2012)

18. Sommer, C., Straehle, C., Köthe, U., Hamprecht, F.A.: Ilastik: interactive learning and segmentation toolkit. In: 2011 IEEE International Symposium on Biomedical Imaging: From Nano to Macro, pp. 230-233 (2011) 